\title{
Risk Factor distribution in patients with Acute Coronary Syndrome
}

\section{Dr. Man Bahadur K. C.}

Coronary Artery disease is emerging as a growing epidemic in whole of the South Asia region. South Asians are genatically most predisposed people to Coronary Artery disease. In this background of genetic predisposition to Coronary Artery disease and changes in life style, which increases the vulnerability to acquire this disease, many Nepalese people are getting Coronary Artery disease with increasing number every year.

There are already well-established risk factors of Coronary Artery disease of which some are modifiable risk factors like smoking, life style and some are not modifiable like, heredity, age and male sex. Presence of major risk factors like smoking, hypertension, diabetes and dyslipidemia, alone or in combination, increases the predisposition to premature and sever CAD.

In this article, an attempt was made to assess distribution of risk factors in patients with Acute Coronary Syndrome (AGS) admitted in Coronary care unit (CCU) of Shahid Gangalal National Heart Centre. The data included the patients who were admitted in CCU of Shahid Gangalal National Heart Centre in between First Kartik 2058 to Last of Aswin 2059. The analysis included a total of 230 patients of which $176(76.50 \%)$ were male and rest were female. 
- NEPALESE HEART JOURNAL •

Table 1: Distribution of Risk Factors in Patients with ACS

\begin{tabular}{|l|l|l|l|}
\hline S.N & Risk Factors & 158 & 68.7 \\
\hline 1 & Smoking & 108 & 47 \\
\hline 2 & Hypertension & 57 & 24.8 \\
\hline 3 & Diabetes Mellitus & 85 & 37 \\
\hline 4 & Dyslipidemia & 24 & 10 \\
\hline 5 & Obesity & 46 & 20 \\
\hline 6 & Family history of CAD & 176 & 76.5 \\
\hline 7 & Age (male>55, female>65 & 157 & 68 \\
\hline 8 & Male Sex & & 24 Patients \\
\hline
\end{tabular}

Table 1: Single/Multiple Risk Factors in Patients with ACS

\begin{tabular}{|l|l|l|}
\hline S.N & Number of Risk Factors & No of Patients \\
\hline 1 & 1 & 11 \\
\hline 2 & 2 & 33 \\
\hline 3 & 3 & 71 \\
\hline 4 & 4 & 63 \\
\hline 5 & 5 & 29 \\
\hline 6 & 6 & 14 \\
\hline 7 & 7 & 5 \\
\hline 8 & 0 & 2 \\
\hline
\end{tabular}


Smoking is a major and independent risk factor of CAD. Smoking increases risk of CAD by 1.6 times, fatal and non-fatal myocardial infarction by 2.3 times, Cessation of smoking significantly reduces both the morbidity and mortality of CAD, nearly by $65 \%$. Smoking acts synergistically with other risk factors of CAD like hypertension, diabetes mellitus, dyslipidemia and oral contraceptives in Increasing the risk of CAD; Dose effect relationship exists in between number of cigarettes consumed per day, duration of smoking, starting age of smoking and odds ratio of CAD. Smoking more than 20 cigarettes per day results 2 to 3 times increased risk of developing CAD. Consumption of as few in 4 cigarettes per day increases the risk of CAD. Tobacco contains more than 10 kinds of pathogenic substances for development of CAD of which Nicotine and Carbon monoxide are two most important substances, Long-term smoking not only enhances the oxidation of LDL-C and reduction of HDL-C, the protective Cholesterol for heart, but also causes the damage to the endothelium, derangement of hemodynamic and coagulation mechanism, increased level of catecholamines in blood. Hypertension Is also independent risk factor of CAD. The risk of development of CAD is parallel to level of risk of diastolic blood pressure, In Hypertensive subjects there is $27 \%$ increased in the risk of CAD, for every $7 \mathrm{~mm} / \mathrm{Hg}$ increase in diastolic blood pressure .

Even if the risk of development of CAD in subjects having upper normal limits of blood pressure level, i.e. Systolic blood pressure $120=139 \mathrm{~mm} / \mathrm{Hg}$ and diastolic blood pressure of $80-90 \mathrm{~mm} / \mathrm{Hg}$ is significantly higher than subjects having systolic blood pressure of less than $120 \mathrm{~mm} / \mathrm{Hg}$ and diastolic blood pressure of less than $80 \mathrm{~mm} / \mathrm{Hg}$. Reduction in diastolic blood pressure of 5 to $6 \mathrm{~mm} / \mathrm{Hg}$ reduces the risk of CAD by 14\%. Diabetes is associated with insulin resistance syndrome and also causes marked impairment of endothelial and smooth muscle cell function and appears to have increased leucocytes adhesion to vascular endothelium, which is a critical early step in the genesis of atheroma. In diabetic subjects, the risk of AMI is $50 \%$ higher in males, and $150 \%$ higher in females. Sudden cardiac death is also $50 \%$ higher in diabetics, Younger diabetes (4564 years) were found to have 3.2 times higher mortality when compared to older group. The diabetes also have three fold to five fold increased rate of future cardiovascular events, the risk is aven higher in diabetic women.

The level of LDL-C has positive correlation with future risk of development of CAD, Because increased level of LDL-C is associated with increased damage of endothelium, which is critical step for atherogenesis. Similarly increased level of HDL-C is negatively correlated with future risk of development of CAD, because HDL-C helps to transfer cholesterol from periphery to liver, which is the main component of an atherosclerotic plaque. 
A number of reports supports that subjects with excess body weight have increased risk of CAD. This may be due to the fact that the obesity may be associated with hyperlipidemia, hypertension and sometimes diabetes mellitus.

Males are more prone to CAD than female and males more than age of 55 and females more than age of 65 have significantly higher chance of developing CAD than subjects with age less than 55 and 65 respectively. Finally, the numbers of risk factors present in subjects with ACS were also reviewed. An interesting trend was observed that subjects with higher number of risk factors; had higher chance of having extensive myocardial infarction, hemodynamic derangement and premature CAD, 